\title{
Behaviour of Non-ideal Saturable Absorber Doped on Active Fiber
}

\author{
Abdallah Ijjeh, Saed Thuneibat \\ Department of Electrical and Electronic Engineering, Al-Balqa`Applied University / Al-Huson University College, Irbid, Jordan \\ Email address: \\ Abdallah.ijjeh.y@gmail.com (A. Ijjeh)

\section{To cite this article:} \\ Abdallah Ijjeh, Saed Thuneibat. Behaviour of Non-ideal Saturable Absorber Doped on Active Fiber. American Journal of Optics and \\ Photonics. Vol. 4, No. 5, 2016, pp. 46-50. doi: 10.11648/j.ajop.20160405.12
}

Received: September 23, 2016; Accepted: October 5, 2016; Published: December 2, 2016

\begin{abstract}
In this paper, a non-ideal saturable absorber doped with an active fiber device is considered. The differential equations that describe the behavior of the device are simplified and solved numerically. The results show that as the non-ideal term (loss) increases, the laser performance degradation increases.
\end{abstract}

Keywords: Multiple Pulsing, Pulsed Lasers, Semiconductor, Saturable Absorbers

\section{Introduction}

In the fiber laser with a non-ideal distributed saturable absorber (FLDSA), two different types of particles are distributed through the fiber core. These two types of particles interact via the electric field in the fiber core $[1,2]$. Different work has been done in FLDSA due to the fact that FLSDA is a simple structure that exhibits laser pulsing behavior, Q-switching, and mode locking in the range of picoseconds [3-11]. In addition, the FLSDA structure is compatible with the existing fiber networks.

Passive mode locking relies on the action of the distributed saturable absorber where its material becomes more transparent to light as the light intensity increases. As a result, if a saturable absorber is placed inside the fiber laser cavity, it produces modulated loss at a separation frequency of $l / c$, where 1 is the fiber length and $\mathrm{c}$ is the speed of light.

In the previous work two different approaches have been used to describe the pulsing behavior under the assumption that the saturable absorber is located as a bulk cell near the end of the fiber and the absorber is an ideal one. In the first approach, the electric field, active medium, and passive medium loss were expanded by Taylor expansion about the maximum to determine the expansion coefficients from the self-consistency condition [7-10]. In the second approach a functional form for the pulse profile was chosen and then its parameters on the basis of a second order approximation was determined $[11,12]$. In this paper the passive mode locking in the non-ideal saturable absorber is described with a mathematical model for pulsing behavior. In this case, the pulse width is usually many orders shorter than the mode separation. A non-ideal saturable absorber is introduced for pulse evaluation, and distributed uniformly in the fiber core thereby reducing the separation frequency between modes. In this approach the three coupled nonlinear differential equations that describe the field inside the fiber, active medium gain, and passive medium loss, are simplified using the slowly varying field approximation. The resulting system is solved numerically.

\section{Mathematical Model}

It is assumed that the active medium is a two level homogeneously broadening system which introduces gain only and distributed uniformly inside the fiber core. The same assumptions apply to non-ideal saturable absorber, except that it introduces attenuation instead of gain with nonideal term. The governing equations that describe the propagation of the time dependent electric field pulse envelope $A(t)$, the active medium gain and the passive medium losses are given by [2]:

$$
\begin{aligned}
& T_{R T} \frac{\partial A(T, \eta)}{\partial T}= \\
& {\left[\left(g+\frac{4 g}{\Delta \omega_{a}^{2}} \frac{d^{2}}{d \eta^{2}}\right)-\left(B-i \delta|A|^{2}\right)-\left(L+i D \frac{d^{2}}{d \eta^{2}}\right)\right] A(T, \eta)}
\end{aligned}
$$

Where $T_{R T}$ is the recovery time for generated pulses, $g$ is 
the gain medium parameter, $\Delta \omega_{a}$ is the bandwidth of the linear resonant filter, $\delta$ is the self-phase modulation and $L$ is the constant fiber losses, including Fresnel losses faces, scattering, and absorption losses within the fiber core (additional losses), and the terms:

$\left(g+\frac{4 g}{\Delta \omega_{a}^{2}} \frac{d^{2}}{d \eta^{2}}\right) A(T, \eta)$, describes the gain medium, while

$\left.(B-i \delta)|A|^{2}\right) A(T, \eta)$, describes the saturable absorber medium with self-phase modulation (SPM), and

$\left(L+i D \frac{d^{2}}{d \eta^{2}}\right) A(T, \eta)$, describes the fiber cavity loses with group velocity dispersion (GVD).

The saturable balanced against gain can be used as a pulse forming mechanism [2].

Let us define $\tau_{S A}$ and $\tau_{\text {sol }}$ as [4]:

$$
\begin{gathered}
\tau_{S A}=\sqrt{\left(8 g / \Delta \omega_{a}^{2}\right) / \sigma A_{0}^{2}}=\frac{16 g}{\sigma \Delta \omega_{a}^{2} P_{a v g} T_{R T}} \\
\left.\tau_{\text {sol }=}=\sqrt{(2|D|} / \delta\left|A_{0}\right|^{2}\right)=4|D| /\left(\delta P_{a v g} T_{R T}\right)
\end{gathered}
$$

to account for the effects of SPM and GVD, and the effects of absorption with gain, respectively.

Due to the fact that $\tau_{S A} \succ \tau_{\text {sol }}$, then the pulse shape is not strongly influenced by the saturation absorber speed; slower saturable absorber can be used to obtain short pulses [2].

The saturable absorber balance against gain can be obtained by dropping the imaginary terms that corresponds to SPM and GVD from equation (1):

$$
T_{R T} \frac{\partial A(T, \eta)}{\partial T}=\left(g+\frac{4 g}{\Delta \omega_{a}^{2}} \frac{d^{2}}{d \eta^{2}}-B-L\right) A(T, \eta)
$$

The gain medium is described by [2]:

$$
\frac{d g(t)}{d t}=\frac{1}{T_{R T}}\left(\frac{I(t)}{I_{1} s a t} g(t)-\left(g_{1}(t)-g_{0}\right)\right)+d \frac{G_{s}(t)}{d t}
$$

Where $g_{O}$ is the small signal gain or totally unsaturated gain, $g_{1}(t)$ is the totally saturated gain, I1 sat is the saturation intensity of the active medium, and $I(t)$ is the light intensity of the output mode locking pulse which can be normalized and written as [2]:

$$
\mathrm{I}(\mathrm{t})=|A(t)|^{2}
$$

The non-ideal saturation absorber can be recognized in the form:

$$
B(t)=\sigma(t)+S_{0}
$$

Where $S_{0}$ is the non-ideal absorption term, and $\sigma(t)$ is the saturable term given by:

$$
\sigma(t)=\frac{\alpha_{0}}{1+I(t) / I_{S A T}}
$$

Then,

$$
B(t)=\frac{\alpha_{0}}{1+I(t) / I_{S A T}}+S_{0}
$$

$\mathrm{G}_{\mathrm{s}}(\mathrm{t})$ is the pump parameter given in [9] by,

$$
\mathrm{Gs}(\mathrm{t})=\mathrm{g} 0 \int_{-\infty}^{t} I_{p}\left(t^{\prime}\right) d t^{\prime}
$$

Where $I_{p}(t)$ is the pump pulse intensity function which is assumed to be in the form:

$$
\mathrm{I}_{\mathrm{p}}(\mathrm{t})=I_{p o} \sec h^{2}\left(\frac{t}{t_{p}}\right)
$$

Where $\mathrm{I}_{p o}$ is the pump pulse amplitude and $\mathrm{t}_{\mathrm{p}}$ is the pump pulse duration.

The gain for active medium far from saturation can be represented by:

$$
g(t)=g_{0}\left(1-\frac{A^{2}(t)}{I_{1 s a t}}\right)-G_{s 1} \sec h^{2}\left(\frac{t}{t_{p}}\right)
$$

where $\mathrm{G}_{\mathrm{s} 1}$ is a new constant equal to $g_{0} T_{R T}$.

Using the slowly varying field approximation for equation (4), and using equations (6-12) the resulting equations after some manipulations can be written as [1-3].

$$
\left(g(t)-L-B(t)+\left(\frac{a_{1}}{\Delta w_{a}}\right) \frac{d}{d t}+\frac{1}{\Delta w_{a}} \frac{d^{2}}{d t^{2}}\right) A(t)=0
$$

Let

$$
\Delta w_{a}\left(g_{0}-L-\alpha_{0}-S_{0}\right)=a
$$

and

Then equation (14) can be rewritten as: 


$$
a A(t)+b A^{3}(t)+a_{1} \frac{d}{d(t)} A(t)+\frac{d^{2}}{d\left(t^{2}\right)} A(t)=\Delta w_{a} G_{s} \sec h^{2}\left(\frac{t}{t_{p}}\right)
$$

Which describes the active fiber with non-ideal absorber. If $R=\Delta w_{a} G_{s}$, then equation (17) can be rewritten as:

$$
a A(t)+b A^{3}(t)+a_{1} \frac{d}{d(t)} A(t)+\frac{d^{2}}{d\left(t^{2}\right)} A(t)=R \sec h^{2}\left(\frac{t}{t_{p}}\right)
$$

Equation (18) can be solved numerically for proper values of media constants, active fiber medium, and non-ideal absorber medium. The nonlinear term in equation (18) involving $A^{3}$ plays an important role in determining the spectral properties of the laser field. However, if $A^{3}$ is neglected, the spectrum of $A(t)$ will correspond to a pumping pulse going through a linear resonant filter with a bandwidth $\Delta \omega_{a}$. This is the case below the threshold where the cubic term can be neglected [14].

\section{Results and Discussions}

The behavior of non-ideal saturable absorber doped on active fiber can be described accurately by equation 18 . This equation accounts for the effects of passive/active fiber mediums, and the pumping characteristics.

Figure 1 shows the typical saturable absorber behavior for ideal and non-ideal absorbers. It is clear that as the irradiance increases, the absorption coefficient decreases. The reduction rate for the non-ideal absorber is less than that for the ideal absorber.

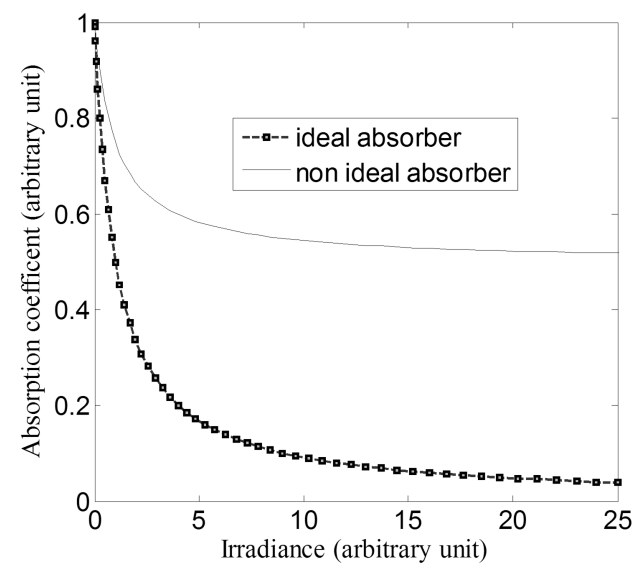

Figure 1. Typical saturable absorber behavior for ideal and non-ideal absorbers.

Figures (2) and (3) show the typical pulses obtained by solving equation (18) for different values of parameters $a$ and $b$ respectively. It is clear that as $a$ or $b$ increases the amplitude and the width of the obtained pulses decrease. The effect of $\alpha_{0}$ and $S_{0}$ on the obtained pulses can be seen clearly from equations (15) and (16). As $\alpha_{0}$ (ideal term) increases, both parameters $a$ and $b$ of equation (18) decreases, however, $S_{0}$ (non-ideal term) affects only parameter $a$.

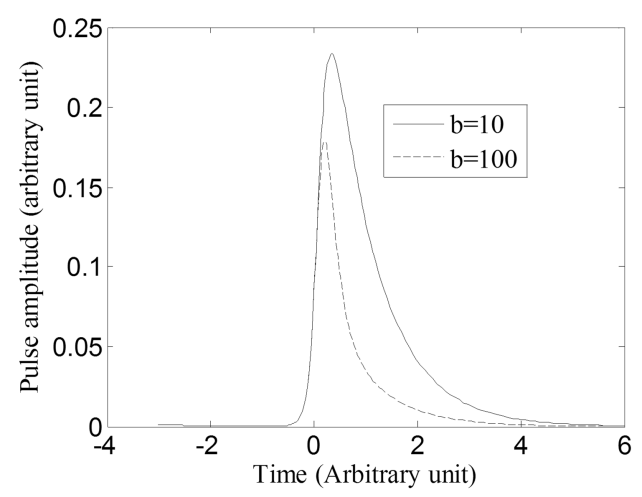

Figure 2. Amplitude of pulse versus time (in arbitrary units). $a=10, R=1000$ and $t_{p}=0.1$.

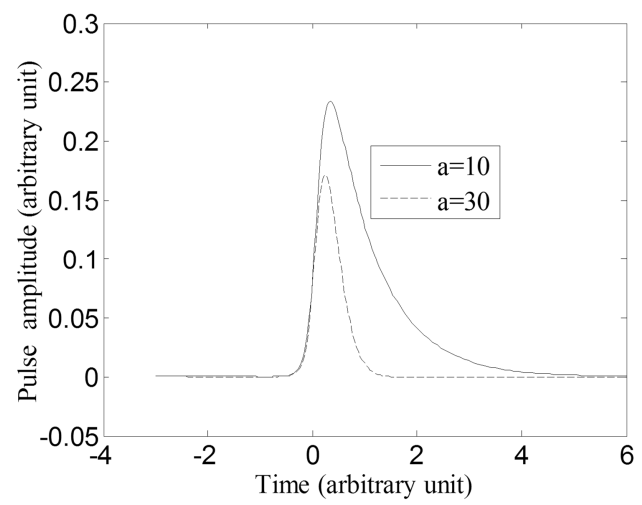

Figure 3. Amplitude of pulse versus time (in arbitrary units) at $b=100$, $R=1000$ and $t_{p}=0.1$.

Figure 4 shows the effect of $R$ on pulsing behavior. As expected, as $R$ increases the pulse amplitude is also increases in the pulsing region, and the pulse width decreases. This approach can be used to generate pulses with small duration.

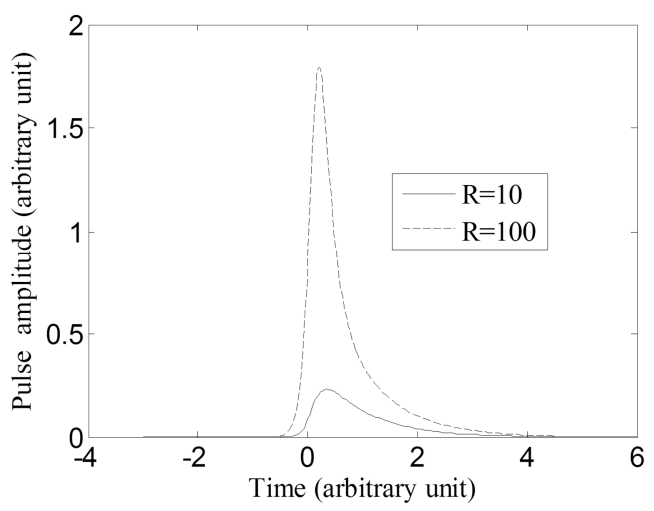

Figure 4. Pulse amplitude versus time (in arbitrary units). $b=10, a=10$ and $t_{p}=0.1$. 
The effect of linear dispersion parameter $a_{1}$ on the side pulses can be clearly seen in figures $5,6,7$ and 8 . As $a_{1}$ decreases, the side pulses increase faster

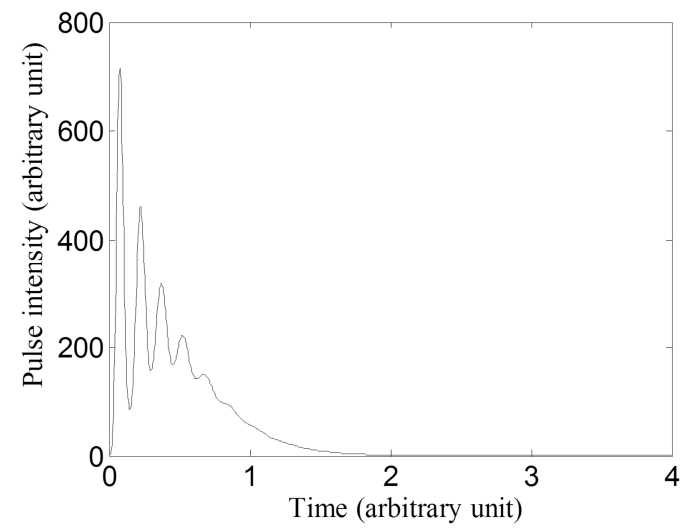

Figure 5. Pulse intensity versus time (in arbitrary units). $a=1000, b=1$, $a_{1}=10, R=150$ and $t_{p}=0.9$.

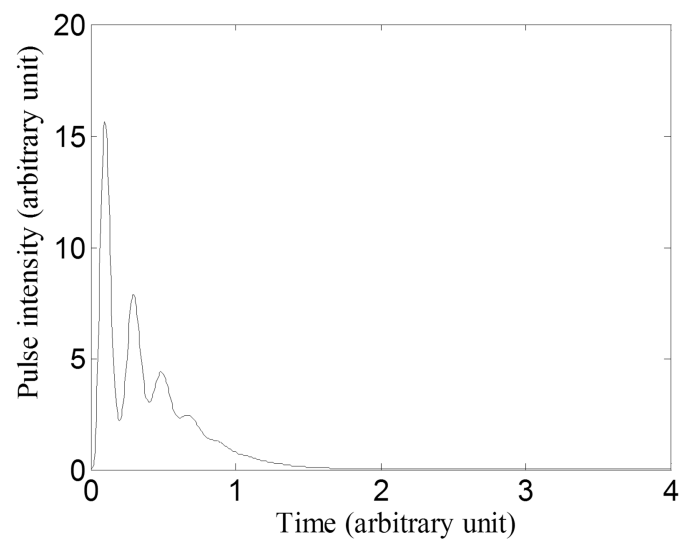

Figure 6. Pulse intensity versus (in arbitrary units). $a=1000, b=1, a_{1}=10$, $R=50$ and $t_{p}=0.9$.

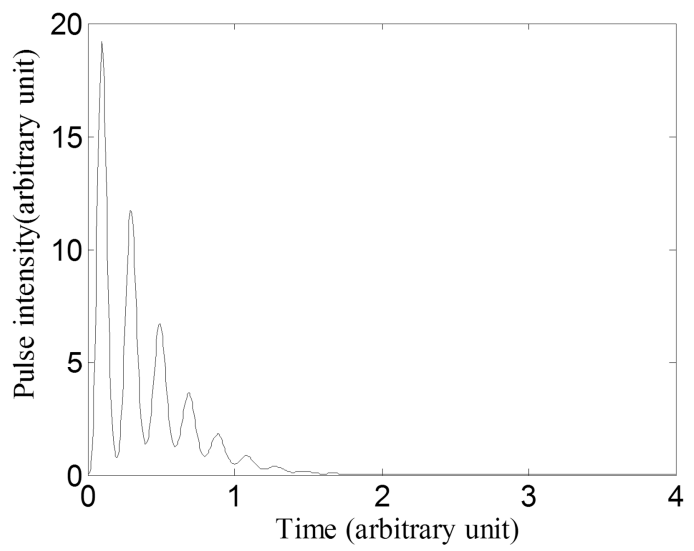

Figure 7. Pulse intensity versus (in arbitrary units). $a=1000, b=1, a_{I}=5$, $R=50$ and $t_{p}=0.9$.

In the absence of $a_{1}$ (see figure 8 ), the side pulses remain approximately fixed with time. Increasing $a_{1}$ is equivalent to increasing the ideal absorber term $\alpha_{0}$.

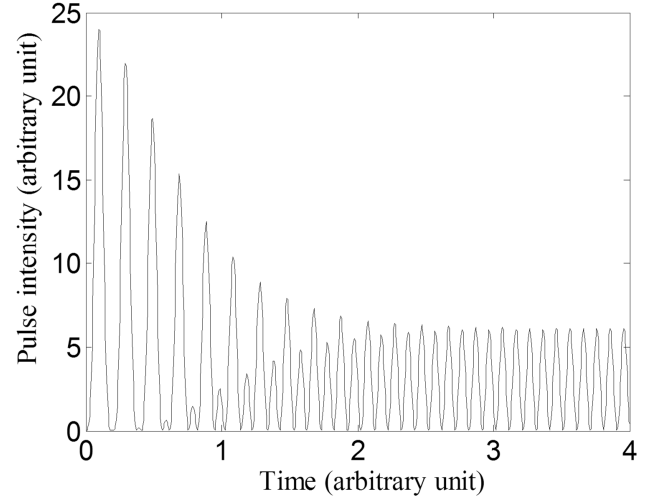

Figure 8. Pulse intensity versus (in arbitrary units). $a=1000, b=1, a_{1}=0$, $R=50$ and $t_{p}=0.9$.

\section{Conclusion}

Using active fiber laser with non-ideal absorber, the behavior of generated pulse is strongly depending on the absorber parameters. As the non-ideal (non-saturable) term increases, the pulse performance degradation increases; pulse amplitude deceases and pulse width increases. The effect of non-saturable parameter and fiber loss on parameter $a$ is the same. This makes the possibility to use high loss fiber with very small non-saturable term or vice versa. By introducing the linear dispersion parameter, the ideal term increases and the effect of non-ideal term is compensated.

\section{References}

[1] Siegman, A. E. (1986). Lasers. University science books, sausalito, California.

[2] Boyd, R. W., Raymer, M. G., Narducci, L. M.. (1986). Optical Instabilities. Cambridge university press, Cambridge.

[3] Keller, U., Weingarten, K. J., Kartner, F. X., Kopf, D., Braun, B., Jung, I. D., Fluck, R., Honninger, C., Matuschek, N., Aus der Au, J. (1996). Semiconductor saturable absorber mirrors (SESAM's) for femtosecondto nanosecond pulse generation in solid-state lasers. IEEE Journal of Selected Topics in Quantum Electronics, 2, 435-453.

[4] Kurtner, F. X., der Au, J. A., Keller, U. (1998). "Mode-locking with slow and fast saturable absorbers-what's the difference?". IEEE Journal of Selected Topics in Quantum Electronics, 4, 159-168.

[5] Ijjeh, A. (1995) Investigation of pulsing operation in Rare earth-doped fiber. proceedings Russian, Telecom StPetersburg. 12-16.

[6] Galkin, S. L. (1991). Fiber optic devices based on Active fibers. Proc. Ist Foc 91. Leningrad. 25-29.

[7] New, G. H. C., Catherall, J. M. (1984). Problems in the selfconsistent profile approach to the theory of laser modelocking. Optics Communications, 50, 111-116.

[8] New, G. C., Zenteno, L. A., Radmre, P. M. (1983). New techniques in the theory of active mode locking. Optics Communications, 48, 149-154. 
[9] Clemens, J. M., Najbar J., Bronstein-Bonte I., Hochstrasser, R. M. (1983). Dual picosecond Dye-lasers, synchronously pumped by A mode locked CW. YAG laser Optics Communications, 47, 271-277.

[10] Graf F., Low, C., Penzkofer A. (1983). Passively mode-locked Nd-glass laser with partially suppressed natural mode selection. Optics Communications, 47, 329-334.

[11] Haus H. A. (1975). Theory of mode locking with a slow saturable absorber. IEEE J. of Quantum Electronics, 11, 736746.

[12] Catherall, J. M., New, G. H. C., Radmore, P. M. (1982). Approach to the theory of mode locking by synchronous pumping," Optics Letters. 7, 319.
[13] Chen, Yao, et al. "Optically driven black phosphorus as a saturable absorber for mode-locked laser pulse generation." Optical Engineering 55.8 (2016).

[14] Yariv A. (1975). Quantum Electronics. John Willy and Sons. Inc

[15] Terrien, Soizic, et al. "Multistable dynamics in a micropillar laser with saturable absorber and delayed optical feedback." Nonlinear Photonics. Optical Society of America, 2016.

\section{Biography}

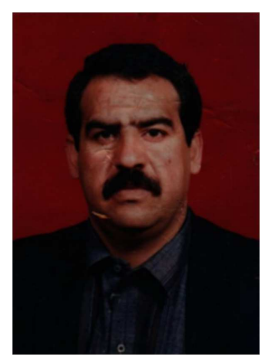

Dr. Abdullah Ijjeh has received his BSc in Electrical Engineering from Kuwait University in 1981. He received his M.Sc. in communication engineering from Yarmouk University, Jordan in 1988. He received his PhD in communication engineering from The Bonch-Bruevich Saint - Petersburg State University of Telecommunications, St. Petersburg, Russia 1995. Currently he is working at the Department of communication engineering at Al-Balqa' Applied University, Jordan. His research interests are fiber optics and wireless and communication systems.

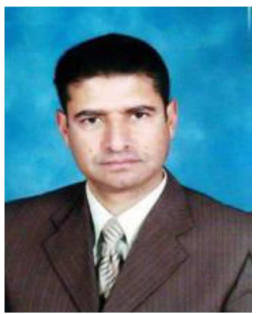

Dr. Saed Thuneibat is currently working as an associate professor at the department of Electrical and Electronic Engineering at the Al-Huson University College, Al-Balqa Applied University, Jordan. He specializes in research and teaching in optical and Digital telecommunication systems, Computer Networks. His research interests include Optical Fiber Performance, Digital Communication Systems and Networks. 